Ar)

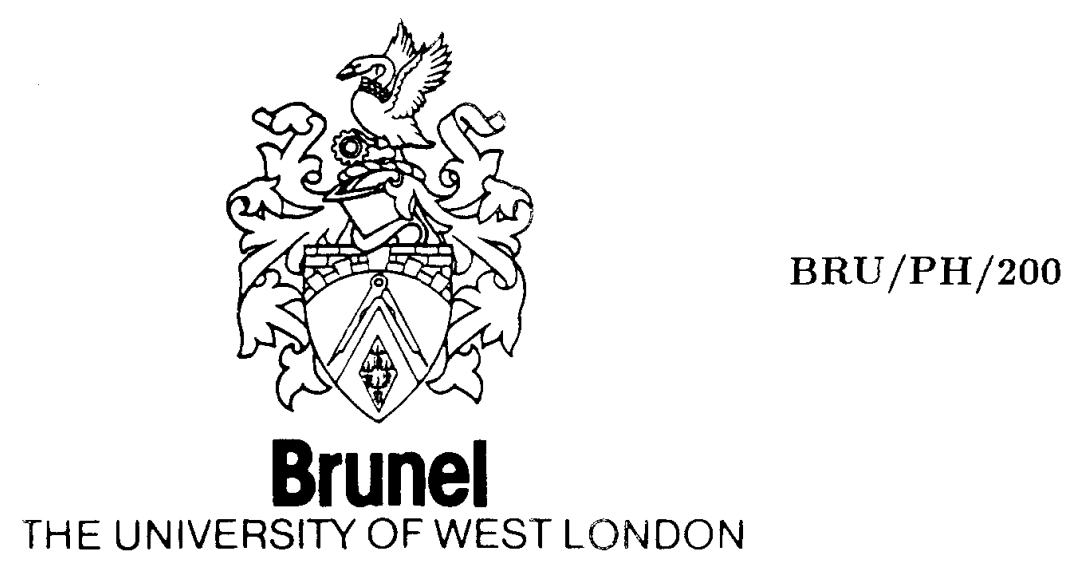

\title{
Spin Effects in Hadroproduction \\ of Charmed Hadrons
}

Robert Ryłko

Department of Physics, Brunel University, Uxbrigde UB8 3PH, United Kingdom.

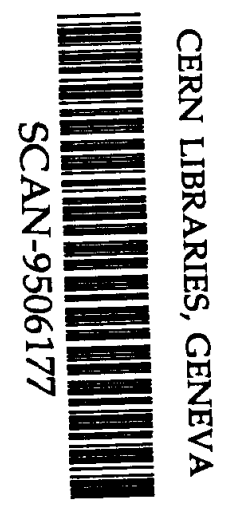

Sw 9526

(Talk presented at the 11th International Symposium on High Energy Spin Physics (SPIN 94), September 15-22, 1994, Bloomington, IN, USA.) 
BRU $/ \mathbf{P H} / 200$

\title{
Spin Effects in Hadroproduction of Charmed Hadrons
}

\author{
Robert Ryłko ${ }^{1}$ \\ Department of Physics, Brunel University, \\ Uxbrigde UB8 $3 P H$, United Kingdom.
}

\begin{abstract}
We review the results on spin effects in the inclusive production of $D^{*+}(2010)$ and $\Lambda_{c}^{+}$based on the CERN NA32 data. The spin alignment parameter of $D^{*+}(2010)$ meson is measured to be $\eta=$ $0.10_{-0.11}^{+0.12} \pm 0.01$. The angular distribution of decay products in the Gottfried-Jackson frame is consistent with the quark model assignment for $\Lambda_{c}^{+}$spin. For $\Lambda_{c}^{+} p_{T}>1.1 \mathrm{GeV} / c$, the product of the $\Lambda_{c}^{+}$transverse polarization and the kaon type asymmetry for $\Lambda_{c}^{+} \rightarrow p K^{-} \pi^{+}$is measured to be $\alpha_{K} P_{\Lambda_{c}}=-0.65_{-0.18}^{+0.22}$ (anti-Basel). The $\Lambda^{0}$ polarization in the same experiment is measured to be $-0.28 \pm 0.09 \pm 0.02$ (Basel), for $\Lambda^{0} p_{T}>1 \mathrm{GeV} / \mathrm{c}$.
\end{abstract}

\section{INTRODUCTION}

Currently, in the charm sector, there are two particles ${ }^{2}$ with nonzero spin for studying spin effects via exclusive decay channels with reasonable statistics. These are $D^{*+}(2010)$ and $\Lambda_{c}^{+}$, with quark model assignments $J^{P}=1^{-}$and $J^{P}=\frac{1}{2}^{+}$, respectively. In both cases the spin measurements are lacking ${ }^{3}$ and studies of spin effects with these particles assume their quark model spin assignment.

This paper reviews the results of spin effects in the inclusive hadroproduction of $D^{*+}(2010)$ and $\Lambda_{c}^{+}$. The results are based on data from the CERN NA32 fixed $C u$ target experiment, using the $230 \mathrm{GeV} / \mathrm{c} \pi^{-}$beam, described elsewhere [Ref.(1)]. In addition, other spin effects involving charmed hadrons are listed.

\footnotetext{
${ }^{1}$ Previously at Queen Mary \& Westfield College, University of London, Mile End Rd., London E1 4NS, United Kingdom.

${ }^{2}$ Throughout the paper a charmed particle symbol stands for particle and antiparticle, i.e. any reference to a specific state implies the charge-conjugate state as well.

${ }^{3}$ In this paper we review the results on the measurement of the $\Lambda_{c}^{+}$spin with the use of the $\Lambda_{c}^{+} \rightarrow p K^{-} \pi^{+}$channel.
} 


\section{THE $D^{*+}(2010)$ MESON [Ref.(2)]}

The $D^{*+}(2010)$ sample consisted of 127 events. Using the same selection criteria 20 wrong sign events were found. The angular distribution for the $D^{*+}(2010) \rightarrow D^{0} \pi_{e x t}^{+}$decay is given by

$$
I(x)=\frac{1}{2}\left[1+d_{2} P_{2}(x)\right] \equiv \frac{1}{2}+\eta P_{2}(x),
$$

where $P_{2}(x)$ is the second Legendre polynomial, $\eta=\left(3 \rho_{00}-1\right) / 2$ is the spin alignment parameter, and the variable $x \equiv \cos \theta_{\text {hel }}$ stands for the cosine of the angle between the $D^{*+}$ direction in the laboratory frame and the $\pi_{e x t}^{+}$direction in the $D^{*+}$ rest frame.

The results of the maximum-likelihood fit, with the background subtracted, for the whole sample is $\eta=0.10_{-0.11}^{+0.12} \pm 0.01$ [Ref.(2)]. In four equally populated subsamples, differing in $x_{F}$ and $p_{T}$ cuts, the spin alignment parameter $\eta$ is consistent with zero.

The relative abundance of vector mesons $P_{V}^{\sigma}=V /(P+V)$, where $P$ and $V$ stands for the cross sections for the production of $D^{+}$and $D^{*+}(2010)$ mesons, may be calculated using $\eta$ within the statistical assumption (namely, for the spin dependent hadronization of the $c$ quark combining with the sea antiquark to form a meson [Ref.(3)]). The $P_{V}$ calculated within this picture

$$
P_{V}^{\eta}=\frac{3}{4(1-\eta)},
$$

should be equal to that from the cross section measurements, i.e. $P_{V}^{\sigma}=P_{V}^{\eta}$.

This simple picture works for a number of different production processes for $\pi^{0} / \rho^{0}$ and $K_{s}^{0} / K^{* 0}$ systems (see Ref.(4) for references). The only other measurements of the spin alignment parameter for the $D^{*+}(2010)$ come from $e^{+} e^{-}$collisions [Refs.(4), (5)]. For charm mesons the $\left(e^{+} e^{-}\right)$average, recalculated using the latest charm branching fractions (see Ref.(2) for details), is $P_{V}^{\sigma}=0.70 \pm 0.07$, which is consistent with $P_{V}^{\eta}=0.77 \pm 0.02 \pm 0.01$ [Ref.(4)]. Hence the $e^{+} e^{-}$results for charm mesons agree well with the statistical approach.

The measured spin alignment $\eta=0.10_{-0.11}^{+0.12} \pm 0.01$ gives $P_{V}^{\eta}=0.83_{-0.10}^{+0.11} \pm$ 0.01 , which is inconsistent with the directly measured values of $P_{V}^{\sigma}$. The NA32 $\sigma\left(D^{+}\right)$and $\sigma\left(D^{*+}\right)$ cross sections yield $P_{V}^{\sigma}=0.47 \pm 0.11$, and similarly using the same branching fractions, one obtains $P_{V}^{\sigma}=0.46$ with a comparable error from the recent measurements of the E653 collaboration in $\pi^{-}$Emuls. interactions at $600 \mathrm{GeV} / \mathrm{c}$ [Ref.(6)].

There is a definite discrepancy between $P_{V}^{\eta}$ and $P_{V}^{\sigma}$ for hadroproduction. A possible explanation is that contrary to the other processes, the hadroproduction of the $D^{*+}(2010)$ proceeds mainly via the decays of higher $D^{* *}$ mesons. 


\section{THE $\Lambda_{c}^{+}$BARYON}

The $\Lambda_{c}^{+}$is the lowest mass charmed baryon and decays weakly. A natural decay channel to study production/decay spin effects for $\Lambda_{c}^{+}$is the two-body channel $\Lambda_{c}^{+} \rightarrow \Lambda^{0} \pi^{+}$. There are interesting results from studies of the decay spin effects, namely the weak-cascade decay of an unpolarized $\Lambda_{c}^{+} \rightarrow \Lambda^{0} \pi^{+} \rightarrow$ $\left(p \pi^{-}\right) \pi^{+}$. A negative asymmetry $\alpha_{\Lambda_{c}}^{N L}$ of $-1.0_{-0.0}^{+0.4}$ and $-0.96 \pm 0.42$ has been observed in this decay by CLEO and ARGUS [Ref.(7)], respectively. A similar cascade was used to measure the decay asymmetry parameter in the semileptonic decay of $\Lambda_{c}^{+} \rightarrow \Lambda^{0} l^{+} \nu_{l}$, giving $\alpha_{\Lambda_{c}}^{S L}$ equal to $-0.89_{-0.11-0.05}^{+0.17+0.09}$ and $-0.91 \pm 0.49$ from CLEO and ARGUS [Ref.(8)], respectively. The branching ratios for the above channels are few times smaller than for $\Lambda_{c}^{+} \rightarrow p K^{-} \pi^{+}$ (being of order of $4 \%$ only). Besides that, the $\Lambda_{c}^{+} \rightarrow p K^{-} \pi^{+}$channel has a much better experimental signature than the above mentioned channels.

In the rest frame of the decaying particle, the angular orientation of the back-to-back two-body decay is specified by one direction only. For the threebody decay, the momenta of the final particles are bound to the decay plane, thus the three-body angular orientation is fixed by two directions that specify the decay plane and the rotation within it. These two directions may be chosen as: the normal to the decay plane and the direction of a final particle, or just the two directions of final particles. Thus, the resulting angular decay distributions have different decay coefficients, depending on the angular parameterization of the final state.

For the spin measurement, and the polarization $\&$ decay asymmetry studies the practically background free sample of $121 \Lambda_{c}^{+} \rightarrow p K^{-} \pi^{+}$events with positive $x_{F}$ of $\Lambda_{c}^{+}$was used (see Refs.(9), (10)).

$$
\text { Testing } \Lambda_{c}^{+} \text {Spin Hypothesis [Ref.(9)] }
$$

The $\cos \theta$ distribution, i.e. the angular decay distribution integrated over the decay plane rotation angle and the azimuthal angle of the first direction $\phi$, has $(2 J+1)$ parameters $\left(d_{l}\right.$ moments, see Ref. $\left.(9)\right)$ for the spin $J$. Choosing the $Z$ direction of the reference frame to be the beam direction (the GottfriedJackson frame) their number is reduced by parity conservation (the $d_{l}$ moments vanish for odd $l$ ). Thus, this frame is suitable for spin measurements with limited data samples, especially for higher spin hypotheses. On the other hand, among the different angular parameterizations of the final state, the one using the normal to the decay plane as the first direction (i.e. $\theta_{n}^{G J}$ and $\phi_{n}^{G J}$, the polar and the azimuthal angle of the normal to the decay plane) appears to have a moderate acceptance dependence.

The experimental distribution of $\cos \theta_{n}^{G J}$ was fit with the corresponding distribution for the $\Lambda_{c}^{+}$spin $J=1 / 2, J=3 / 2$ and $J=5 / 2$ hypotheses [Ref.(9)]. The results of the maximum-likelihood fits for higher nonvanishing moments are consistent with zero, both for the whole sample and for the subsample of 
$p_{T}\left(\Lambda_{c}^{+}\right)>0.7 \mathrm{GeV} / \mathrm{c}$. It should be recalled here that the range of $d_{2}$ values for $J=3 / 2$ is $[-1,1]$ and for both $d_{2}$ and $d_{4}$, in the case of $J=5 / 2$, is even wider. The integrated distribution in the azimuthal angle $\phi_{n}^{G J}$ is essentially flat, both for the total sample and the $p_{T}>0.7 \mathrm{GeV} / \mathrm{c}$ subsample. Its coefficients $S_{m}^{ \pm}$, which do not vanish identically for $J=1 / 2$ or $J=3 / 2$, are consistent with zero, within the statistical error of 0.13 for the whole sample and of 0.19 for the $p_{T}>0.7 \mathrm{GeV} / \mathrm{c}$ subsample.

\section{$\Lambda_{c}^{+}$Polarization and Decay Asymmetry [Ref.(10)]}

The $\Lambda_{c}^{+}$polarization was studied with respect to the normal to the production plane, within the anti-Basel convention $\vec{p}_{\Lambda_{c}} \times \vec{p}_{\text {beam }}$ used in Ref.(10). Assuming the $J=1 / 2$ spin assignment for the $\Lambda_{c}^{+}$, the integrated distribution of the polar angle of the first direction reads

$$
I\left(\cos \theta_{i}^{T}\right)=\frac{1}{2}\left[1+d_{1}^{i} \cos \theta_{i}^{T}\right] \equiv \frac{1}{2}\left[1+\alpha_{i} P_{\Lambda_{c}} \cos \theta_{i}^{T}\right],
$$

where $P_{\Lambda_{c}}$ is the $\Lambda_{c}^{+}$polarization with respect to the normal to the production plane and $\alpha_{i}$ are the decay asymmetry parameters depending on the choice of the angular parameterization of the final state. The $\theta_{n}^{T}, \theta_{p}^{T}, \theta_{\pi}^{T}$ and $\theta_{K}^{T}$ refer, respectively, to the angle between the normal to the production plane and: the normal to the decay plane, the proton, the pion and the kaon direction.

The results of maximum-likelihood fits of the $d_{1}^{p}$ and $d_{1}^{\pi}$ are consistent with zero, regardless of $p_{T}$. The $d_{1}^{n}$ is consistent with zero within two standard deviations for $\Lambda_{c}^{+} p_{T}>1.1 \mathrm{GeV} / \mathrm{c}$. For the distribution of $\cos \theta_{K}^{T}$ and $p_{T}>1.1$ $\mathrm{GeV} / \mathrm{c}$, the product of the $\Lambda_{c}^{+}$polarization and the kaon type $\Lambda_{c}^{+} \rightarrow p K^{-} \pi^{+}$ asymmetry is measured to be $\alpha_{K} P_{\Lambda_{c}}=-0.65_{-0.18}^{+0.22}$. The systematic error estimated by varying the acceptance within one standard deviation is of order of 0.02 , for high $p_{T}$ fits. Assuming the suggestion in Ref.(11) for the $\Lambda_{c}^{+} \rightarrow$ $p K^{-} \pi^{+}$decay and the heavy quark effective theory predictions and first results [Refs.(7), (8)], the kaon type decay asymmetry should be negative, thus the $\Lambda_{c}^{+}$polarization should be positive (negative) within the anti-Basel (Basel) convention and of order of 0.6-0.8.

\section{Comparison of $\Lambda_{c}^{+}$and $\Lambda^{0}$ Polarization [Ref.(12)]}

There is a different production mechanism for $\Lambda_{c}^{+}$and $\Lambda^{0}$. For positive $x_{F}$, the measured $\mathrm{N}\left(\Lambda_{c}^{+}\right) / \mathrm{N}\left(\Lambda_{c}^{-}\right)=0.99 \pm 0.16$, while $\mathrm{N}\left(\Lambda^{0}\right) / \mathrm{N}\left(\bar{\Lambda}^{0}\right)=1.27 \pm 0.03$ [Refs.(1), (12)]. Thus, the $\Lambda_{c}^{+}$is expected to be oppositely polarized to the $\Lambda_{c}^{-}$ and, neglecting the CP violation in the decay, the angular decay distributions look the same for both. This is not the case with the hyperon, where the hadronization effects have more impact on the light $s$ quark. Nevertheless, it is interesting to compare the polarization of the two baryons in the same production process. 
The hyperon polarization was studied with respect to the normal to the production plane, with the more widely used Basel convention $\vec{p}_{\text {beam }} \times \vec{p}_{\Lambda}$, for a very clean sample of $27217 \Lambda$ hyperons produced in $230 \mathrm{GeV} / \mathrm{c}^{-} \mathrm{Cu}$ interactions [Ref.(12)]. For negative $x_{F}$ there is basically low $p_{T}$ data, thus the measured polarization is consistent with zero. For $x_{F}>0$ and $p_{T}>1 \mathrm{GeV} / \mathrm{c}$ we find $\mathrm{P}\left(\Lambda^{0}\right)=-0.28 \pm 0.09$ (stat.) \pm 0.02 (syst.).

\section{CONCLUSIONS}

We have shown that interesting spin effects arise for inclusive $D^{*+}(2010)$ and $\Lambda_{c}^{+}$hadroproduction. The spin effects in $D^{*+}(2010)$ hadroproduction do not support the statistical picture of spin dependent $c$ quark hadronization, possibly indicating the importance of higher resonance contribution to the $D^{*+}(2010)$ hadroproduction. The $\Lambda_{c}^{+} \rightarrow p K^{-} \pi^{+}$decay channel has been shown to be useful for the $\Lambda_{c}^{+}$spin measurements and the polarization \& decay asymmetry measurements. Evidence has been shown for the high $p_{T} \Lambda_{c}^{+}$ transverse polarization and the kaon type $\Lambda_{c}^{+} \rightarrow p K^{-} \pi^{+}$asymmetry.

\section{ACKNOWLEDGMENTS}

I would like to thank the Royal Society of London and the organizers of the SPIN'94 conference for financial support.

\section{REFERENCES}

(1). ACCMOR coll., Barlag S., et al., Phys. Lett. B218(1989)374, B247(1990)113, B278(1992)480.

(2). Rybicki K., Ryłko R., Acta Phys. Pol. B24(1993)1049.

(3). Donoghue J.F., Phys. Rev. D19(1979)2806.

(4). CLEO coll., Kubota Y., et al., Phys. Rev. D44(1991)593.

(5). HRS coll., Abachi S., et al., Phys. Lett. B199(1987)585; TPC/Two-Gamma coll., Aihara H., et al., Phys. Rev. D43(1991)29.

(6). E653 coll., Kodama K., et al., Phys. Lett. 284(1992)461.

(7). CLEO coll., Avery P., et al., Phys. Rev. Lett. 65(1990)2842; ARGUS coll., Albrecht H., et al., Phys. Lett. B274(1992)239.

(8). CLEO coll., Bergfeld T., et al., Phys. Lett. B323(1994)219; ARGUS coll., Albrecht H., et al., Phys. Lett. B326(1994)320.

(9). Jeżabek M., Rybicki K., Ryłko R., Acta Phys. Pol. B23(1992)771.

(10). Jeżabek M., Rybicki K., Ryłko R., Phys. Lett. B286(1992)175.

(11). Bjorken J.D., Phys. Rev. D40(1989)1513.

(12). ACCMOR coll., Barlag S., et al., Phys. Lett. B325(1994)531. 